\title{
Free float and stochastic volatility: the experience of a small open economy
}

\author{
Faruk Selçuk* \\ Department of Economics, Bilkent University, Bilkent 06800, Ankara, Turkey
}

Received 23 March 2004; received in revised form 18 April 2004

Available online 26 June 2004

\begin{abstract}
Following a dramatic collapse of a fixed exchange rate based inflation stabilization program, Turkey moved into a free floating exchange rate system in February 2001. In this paper, an asymmetric stochastic volatility model of the foreign exchange rate in Turkey is estimated for the floating period. It is shown that there is a positive relation between the exchange return and its volatility. Particularly, an increase in the return at time $t$ results in an increase in volatility at time $t+1$. However, the effect is asymmetric: a decrease in the exchange rate return at time $t$ causes a relatively less decrease in volatility at time $t+1$. The results imply that a central bank with a volatility smoothing policy would be biased in viewing the shocks to the exchange rate in favor of appreciation. The bias would increase if the bank is also following an inflation targeting policy.

(c) 2004 Elsevier B.V. All rights reserved.
\end{abstract}

Keywords: Exchange rates; Stochastic volatility; Leverage effect; Free float; Turkey

\section{Introduction}

Following a dramatic collapse of an IMF backed, fixed exchange rate based inflation stabilization program, Turkey moved into a free floating exchange rate system in February 2001. Since then, the Central Bank of Turkey has insisted that the monetary authority would stick with the floating exchange rate regime and pursue an implicit inflation targeting policy by controlling the monetary aggregates along with an indicative interest rate. The Central Bank has asserted several times that the level or the direction

\footnotetext{
* Tel.: +90-532-294-8796; fax: +1-208-694-3196.

E-mail address: faruk@bilkent.edu.tr (F. Selçuk).

URL: http://www.bilkent.edu.tr/ faruk.
} 

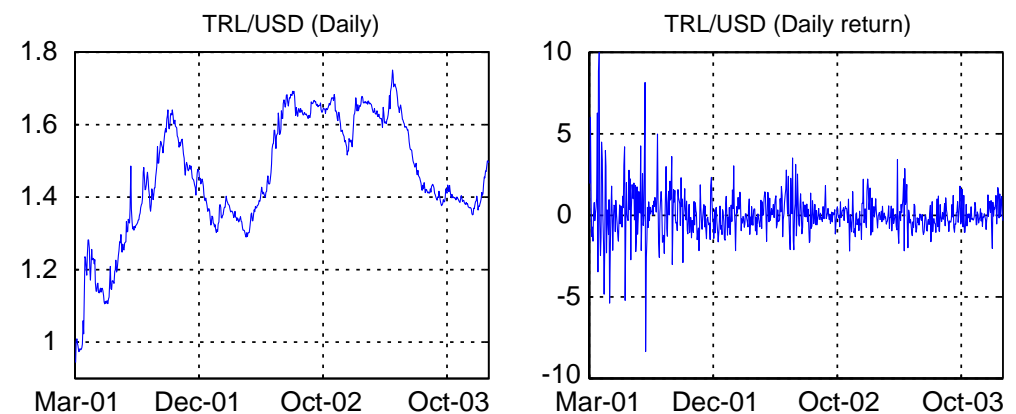

(a)

(b)

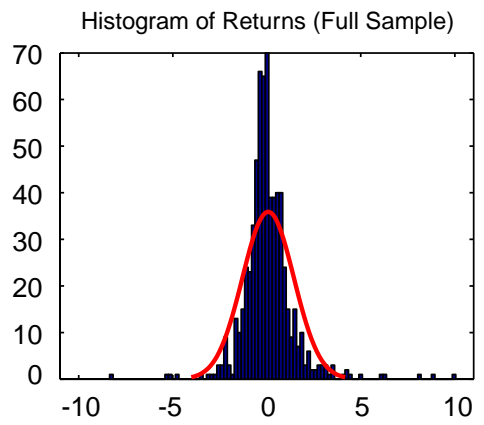

(c)

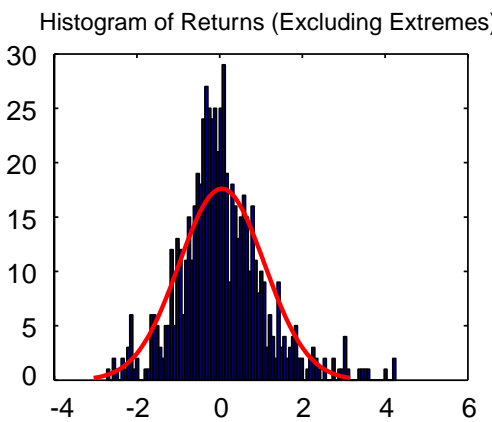

(d)

Fig. 1. TRL/USD daily exchange rate during the floating exchange rate regime in Turkey. (a) The level (in million TRL). (b) Continuously compounded daily return of TRL/USD (percent) (c) Histogram of daily returns. (d) Histogram of daily returns excluding 1\% of extremes from both tails. Sample period: March 12 , 2001-October 30, 2003, covering 667 business days. Data source: the Central Bank of Turkey.

of nominal exchange rates has not been a target but the volatility of the exchange rate is a real concern (CBT [1]).

At the beginning of the float period, the daily change in exchange rate fluctuated wildly. The Central Bank's policies on the one hand and the developments in the supply side of the exchange rate market along with favorable external factors on the other hand resulted in a decrease in volatility and the exchange rate is relatively stabilized (see Fig. 1). ${ }^{1}$ The initial sharp real depreciation of the Turkish Lira (TRL) against major currencies was reversed later and the real appreciation reached to record levels, especially during the first three quarters of 2003. According to the real effective exchange rate index of the Central Bank, the overall real appreciation between February 2001 and September 2003 is $44 \%$.

Substantial appreciation of TRL can be explained to a certain extent by a sharp increase in labor productivity and a fall in real wages during the same period. The labor productivity in the private manufacturing industry went up $12 \%$ between the last

\footnotetext{
${ }^{1}$ See, Selçuk [2] and references therein for a detailed account of the developments in the Turkish economy during the floating exchange rate period of February 2001-August 2003.
} 
quarter of 2000 and the second quarter of 2003, accompanied by a fall in nominal wages in USD terms. As a result, the unit wage index in the private manufacturing industry (in USD) decreased $21 \%$ which partially justifies the real appreciation. However, although there was an upward correction in nominal exchange rates in October 2003, the currency is still over appreciated by any means of comparison and the dynamics behind the record level of real appreciation should be investigated.

In this paper, an asymmetric stochastic volatility model of the foreign exchange rate in Turkey is estimated. It is shown that there is a positive and strong relation between the exchange rate return and its volatility. Particularly, an increase in the exchange rate return (nominal depreciation) at time $t$ results in an increase in its volatility at time $t+1$. However, the effect is asymmetric: a decrease in the exchange rate return at time $t$ causes a relatively less decrease in volatility at time $t+1$. The result implies that the Central Bank would be biased in viewing the shocks to the foreign exchange rate in favor of appreciation. In other words, the volatility smoothing policy of the Central Bank might be a significant contributing factor in the real appreciation of the currency even if the foreign exchange rate system is officially a free-float.

The paper is structured as follows. The next section introduces the asymmetric stochastic volatility model and its implications in a foreign exchange rate market framework. Section 3 reports the estimation method and results. The same section contains a discussion of the estimation results. We conclude afterwards.

\section{Asymmetric volatility}

The stochastic volatility (SV) approach models the volatility as a latent process and it is commonly used in finance literature, especially in option pricing. See, for example, Hull and White [3] and the survey article by Ghysels et al. [4].

Consider the following differential equations describing the dynamics of a stock price $S$ and its volatility $\sigma$

$$
\begin{aligned}
& \mathrm{d} \ln S(t)=\sigma(t) \mathrm{d} B_{1}(t), \\
& \mathrm{d} \ln \sigma^{2}(t)=\alpha+\beta \ln \sigma^{2}(t) \mathrm{d} t+\sigma_{v} \mathrm{~d} B_{2}(t),
\end{aligned}
$$

where $B_{1}(t)$ and $B_{2}(t)$ are two correlated Brownian motions with $\operatorname{corr}\left(\mathrm{d} B_{1}(t), \mathrm{d} B_{2}(t)\right)=$ $\rho$. When $\rho<0$, the price of the asset and its volatility are negatively correlated. One explanation is that a decrease in the asset price increases the debt-equity ratio of the firm and the risk associated with the firm increases. This is known as "leverage effect" in the literature (Black [5]). The discrete version of the model can be stated as

$$
\begin{aligned}
& r_{t}=\sigma_{t} u_{t}, \\
& \ln \sigma_{t+1}^{2}=\alpha+\phi \ln \sigma_{t}^{2}+\sigma_{v} v_{t+1},
\end{aligned}
$$

where $r_{t}=\ln \left(S_{t+1} / S_{t}\right)$ is a continuously compounded rate of return on asset $S(t), \phi=$ $1-\beta$ and $\sigma_{v}$ is the volatility of the volatility. In this representation, $u_{t}=B_{1}(t+1)-B_{1}(t)$ 
and $v_{t}=B_{2}(t)-B_{2}(t-1)$ are independent Gaussian white noises with $\operatorname{corr}\left(u_{t}, v_{t+1}\right)=\rho .^{2}$ Notice that because of the Brownian motion assumption, the standard deviation of the asset return is assumed to be an indicator of the risk associated with the firm. As shown by Meyer and $\mathrm{Yu}$ [6], the following nonlinear state space form shows the role played by the correlation coefficient $\rho$ :

$$
\begin{aligned}
& r_{t}=\sigma_{t} u_{t}, \\
& \ln \sigma_{t+1}^{2}=\alpha+\phi \ln \sigma_{t}^{2}+\sigma_{v} \sigma_{t}^{-1} \rho r_{t}+\sigma_{v} \sqrt{1-\rho^{2}} w_{t+1},
\end{aligned}
$$

where $w_{t+1}=\left(v_{t+1}-\rho u_{t}\right) / \sqrt{1-\rho^{2}}$. Clearly, a unit increase in stock return at time $t$ results in a $\sigma_{v} \sigma_{t}^{-1} \rho$ unit change in the logarithm of the variance at time $t+1$. If the correlation coefficient $\rho$ is negative, a unit fall in stock return at this period will result in $\mathrm{e}^{\sigma_{v} \sigma_{t}^{-1} \rho}$ unit increase in the variance at time $t+1$. For example, suppose that $\rho=-0.60, \sigma_{v}=0.15$, and $\sigma_{t}=1$. If the stock return falls $5 \%$ at time $t$, there will be $25 \%$ increase in the standard deviation next period. However, if there is a $5 \%$ increase in the stock return, the corresponding decrease in volatility will be $20 \%$. Hence, the model implies an asymmetric effect of return on volatility. Notice that for a given pair of $-\rho$ and $\sigma_{v}$, the higher the shock to return process $r_{t}$ is, the more asymmetric the effect on volatility $\sigma_{t+1}$. On the other hand, the pair of $-\rho$ and $\sigma_{v}$ together determines the effect of a given change in stock return on the volatility. Therefore, even if the absolute value of the correlation coefficient $\rho$ is small, a relatively high value of the volatility of the volatility $\sigma_{v}$ may cause a significant asymmetric effect.

Asymmetric stochastic volatility and the case for a leverage effect is less clear for exchange rates. In a small open economy, it is customary to assume that a nominal depreciation (an increase in the exchange rate defined as the domestic currency price of foreign exchange) increases the debt burden of the country on impact. As a result, the country as a whole (and its currency) becomes riskier than before and the risk associated with that country increases. In terms of Eqs. (3) and (4), this means that the correlation coefficient between shocks to the exchange rate return at time $t$ and shocks to its volatility (as a measure of the country and currency risk) at time $t+1$ is expected to be positive. The magnitude of the correlation coefficient $\rho$, along with the volatility of the volatility coefficient $\sigma_{v}$ will capture the asymmetric nature of return-volatility relation in the foreign exchange market.

\section{Estimation and discussion}

The daily continuously compounded TRL/USD return is defined as

$$
r_{t}=\left(\log x_{t}-\log x_{t-1}\right) \times 100, \quad t=1,2, \ldots, 667,
$$

where $x_{t}$ is the indicative TRL/USD exchange rate, measured from the market transaction data during the day by the Central Bank. The sample period is March 12, 2001-

\footnotetext{
${ }^{2} \mathrm{Yu}$ [7] points out an important difference between assuming $\operatorname{corr}\left(u_{t}, v_{t+1}\right)=\rho$ as above and $\operatorname{corr}\left(u_{t}, v_{t}\right)=\rho$ as in Jacquier et al. [8]. Since the specification of Jacquier et al. [8] makes the leverage effect ambiguous, we follow $\mathrm{Yu}[7]$ in our specification.
} 
October 30, 2003, covering 667 business days. The sample statistics indicate that the return series has a large standard deviation (1.36) as compared to the mean (0.074), and the return distribution is skewed to the right. Even if we exclude $1 \%$ of extremes from both tails, the sample skewness falls only from 1.27 to 0.66 (see histograms in Fig. 1).

Stochastic volatility models can be estimated using different approaches. For example, Harvey and Shephard [9] estimates the model with quasi maximum likelihood method. Another approach is Markov Chain Monte Carlo (MCMC) method which was introduced by Jacquier et al. [10] and later extended by Jacquier et al. [8]. Meyer and $\mathrm{Yu}$ [6] and $\mathrm{Yu}$ [7] also utilize MCMC approach using the software Bayesian inference using Gibbs sampling (BUGS). ${ }^{3} \mathrm{Yu}$ [7] gives an alternative representation of Eqs. (5) and (6) by specifying the state and observation equations as follows ${ }^{4}$

$$
\begin{aligned}
& h_{t+1} \mid h_{t}, \alpha, \phi, \sigma_{v}^{2} \sim N\left(\alpha+\phi h_{t}, \sigma_{v}^{2}\right), \\
& r_{t} \mid h_{t}, h_{t+1}, \alpha, \phi, \sigma_{v}^{2}, \rho \sim N\left(\frac{\rho}{\sigma_{v}} \mathrm{e}^{h_{t} / 2}\left(h_{t+1}-\alpha-\phi h_{t}\right), \mathrm{e}^{h_{t}}\left(1-\rho^{2}\right)\right) .
\end{aligned}
$$

In our estimations, we employ the MCMC approach and utilize the code provided by $\mathrm{Yu}$ [7] for the BUGS software. ${ }^{5}$ In setting the prior distributions, we follow Kim et al.

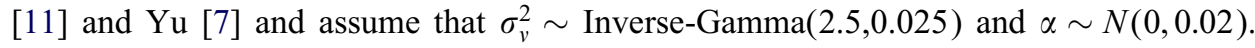
The first prior ensures that the volatility of volatility is positive and relatively low. The second prior indicates that the volatility process may have a constant term. As we have no prior information on this parameter, the mean of the normal distribution is assumed to be zero. Regarding the persistency parameter $\phi$, we could have specified a flat prior $\pi(\phi) \propto 1$. However, this prior is problematic when the data are close to be non-stationary (see Kim et al. [11] and references therein). Therefore, we assume $\phi^{*} \sim \beta(20,1.5)$ where $2 \phi^{*}-1=\phi$, which implies a prior mean of 0.86 for $\phi$. The Beta distribution assumption reflects our belief that the persistency parameter $\phi$ is close to but below unity. In other words, we assign a higher probability of stationarity. Finally, the correlation coefficient $\rho$ is assumed to be uniformly distributed between -1 and 1 , explicitly showing that we have no information prior on this parameter. ${ }^{6}$

The MCMC sampler is initialized by setting $\phi=0.98, \sigma_{v}^{2}=0.03$, and $\rho=0.50$. During our initial analysis, we run 3 chains simultaneously and investigated the trace plot after 20,000 iterations (see Fig. 2, column 1). The results indicated that all chains quickly converged to the same stationary distributions for all parameters. Furthermore, we replicated this analysis by changing the initials to make sure that the result were

\footnotetext{
${ }^{3}$ BUGS (Bayesian inference using Gibbs sampling) is a piece of computer software for the Bayesian analysis of complex statistical models using Markov Chain Monte Carlo methods. The software is freely available at http://www.mrc-bsu.cam.ac.uk/bugs/welcome.shtml.

${ }^{4}$ The model can be modified to capture possible fat-tails in the mean equation innovation $u_{t}$ by changing the normality assumption. See Refs. [6,8] more on this.

${ }^{5}$ BUGS code used in our MCMC estimations of the parameters can be downloaded from Professor Jun Yu's web site http://www.mysmu.edu/faculty/yujun/research.html.

${ }^{6} \mathrm{We}$ experimented with some other prior settings for $\sigma_{v}^{2}$ and $\phi^{*}$ by changing the prior distribution parameters. The Markov chain converged to the same stationary distributions for all parameters.
} 

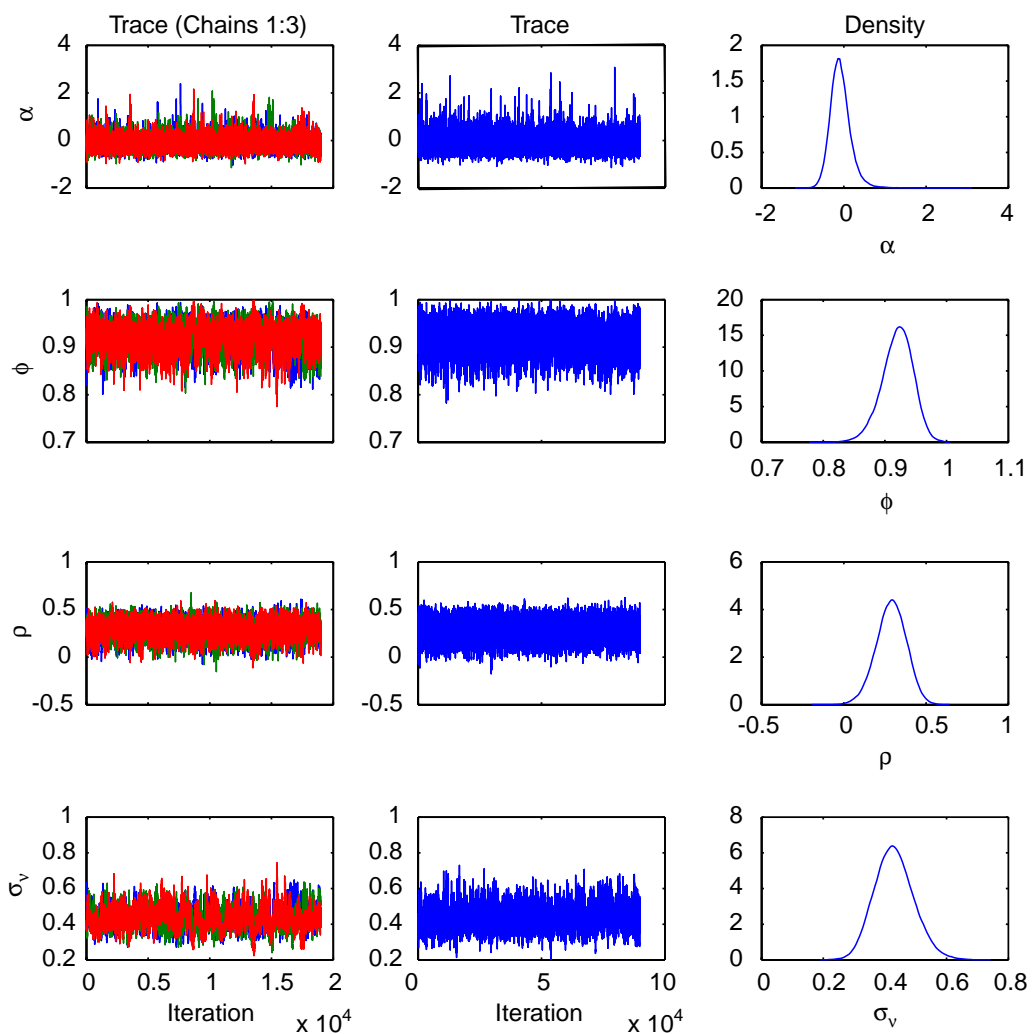

Fig. 2. Traces and kernel density estimates of the marginal posterior distribution of model parameters: From top to bottom raw: $\alpha, \phi, \rho$, and $\sigma_{v}$. The posterior means of parameters are given in Table 1 . The first column shows the estimated parameter values from 3 different chains with a 20000 iterations after a 1000 "burn-in" period. Clearly, the estimates from different chains converge very fast. The second column plots estimated parameters from a single chain with 90000 iterations after an 11000 "burn-in" period. The kernel density estimates (the last column) are based on the single chain estimation.

not sensitive to our initial assumption. There was no significant change in posterior distributions. We therefore used a burn-in of 11000 out of 101000 cycle single chain and used the remaining 90000 to obtain the final posterior means of coefficients and credibility intervals. The chain passed the Heidelberger-Welch stationarity test and lags and autocorrelations were low for all parameters. ${ }^{7}$ Posterior means of parameter estimates along with $95 \%$ posterior credibility intervals are presented in Table 1 . Traces and kernel density estimates of the marginal posterior distribution of model parameters are plotted in Fig. 2. The first column shows the estimated parameter values from 3 different chains with a 20000 iterations after a 1000 "burn-in" period. Clearly, the

\footnotetext{
${ }^{7}$ The CODA software is utilized in diagnostic calculations. See Best et al. [12] for more information on the software and these convergence diagnostics.
} 
Table 1

MCMC parameter estimates of stochastic volatility model in Eqs. (5) and (6)

\begin{tabular}{lllll}
\hline$\alpha$ & $\phi$ & $\rho$ & $\sigma_{v}$ & $\rho \sigma_{v}$ \\
\hline-0.080 & 0.920 & 0.288 & 0.430 & 0.124 \\
{$\left[\begin{array}{llll}-0.514 & 0.487\end{array}\right]$} & {$\left[\begin{array}{lll}0.866 & 0.966\end{array}\right]$} & {$\left[\begin{array}{lll}0.103 & 0.460\end{array}\right]$} & {$\left[\begin{array}{lll}0.313 & 0.563\end{array}\right]$} & {$\left[\begin{array}{ll}0.032 & 0.259\end{array}\right]$} \\
\hline
\end{tabular}

$95 \%$ posterior credibility intervals are given in brackets under each coefficient. These estimates are based on a single chain with 90000 iterations after an 11000 "burn-in" period.

estimates from different chains converge very fast. The second column plots the estimated parameters from a single chain with 90000 iterations after an 11000 "burn-in" period. The kernel density estimates of the marginal posterior distribution of model parameters in the last column are based on this single chain estimations.

The posterior mean of the correlation coefficient $\hat{\rho}$ is positive $(0.29)$ and the credibility interval contains only positive values. The mean of the volatility of volatility coefficient $\hat{\sigma}_{v}$ is 0.43 with a credibility interval of 0.31 and 0.56 . The posterior means of these parameters together indicate that an increase in the exchange rate return $r_{t}$ at time $t$ increases the volatility at time $t+1$. For example, suppose that $\sigma_{t}=1$. If the exchange rate increases $5 \%$ today, the volatility would increase $36.2 \%$ tomorrow. However, if the exchange rate falls $5 \%$, the corresponding fall in the volatility would be only $26.6 \%{ }^{8}$ Hence, a Central Bank with a concern only about the high volatility would respond to shocks to the exchange rate differently. Furthermore, if the Central Bank has an implicit low inflation targeting among its policy objectives, the bias would increase in favor of negative shocks to the exchange rate since there is usually a pass-through from exchange rates to domestic prices.

\section{Conclusion}

Asymmetric stochastic volatility and the case for a leverage effect is less clear for exchange rates. This paper provides evidence for a positive relation between the foreign exchange return and its volatility during a floating exchange rate regime experience of a small open economy. The estimation results of an asymmetric stochastic volatility model indicate that an increase in the foreign exchange return results in an increase in its volatility during the next period. However, the estimated effect is asymmetric: a decrease in the exchange rate return does not result in a decrease in volatility as much. The result implies that a central bank with a volatility smoothing policy might be biased in viewing shocks to the foreign exchange rate in favor of appreciation. The bias would increase if the central bank has an implicit inflation targeting policy. The study can be extended to cover a larger sample of small economies with free floating exchange rate systems versus developed market economies to recover any differences or similarities in exchange rate dynamics.

\footnotetext{
${ }^{8}$ Increase in volatility $\sigma_{t+1}$ is calculated as $\mathrm{e}^{1 / 2(0.288)(0.43)(5)}=1.362$ whereas the fall is given by $\mathrm{e}^{1 / 2(0.288)(0.43)(-5)}=0.734$.
} 


\section{Acknowledgements}

Financial support from the Research Development Grant Program of Bilkent University is gratefully acknowledged.

\section{References}

[1] CBT. General framework of the monetary and exchange rate policy in 2003, The Central Bank of the Republic of Turkey, Basic Policy Readings, 2003, http://www.tcmb.gov.tr.

[2] F. Selçuk, The policy challenge at floating exchange rates: Turkey's recent experience, Mimeo. Bilkent University, Department of Economics, Ankara, Turkey, 2003.

[3] J. Hull, A. White, Hedging and risks from writing foreign currency options, J. Int. Money Finance 6 (1987) 131-152.

[4] E. Ghysels, A. Harvey, E. Renault, Stochastic volatility, in: G.S. Maddala, C.R. Rao, H.D. Vinod, (Eds.), Handbook of Statistics, Vol. 14, North-Holland, Amsterdam, 1996.

[5] F. Black, Studies of stock price volatility changes. in: Proceedings of the 1976 Meetings of the Business and Economic Statistics Section, American Statistical Association, 1976, pp. 177-181.

[6] R. Meyer, J. Yu, BUGS for a Bayesian analysis of stochastic volatility models, Econometrics J. 3 (2000) 198-215.

[7] J. Yu, MCMC methods for estimating stochastic volatility models with leverage effects: comments on Jacquier, Polson and Rossi (2002), Mimeo. The University of Auckland, Department of Economics, Auckland, New Zeland.

[8] E. Jacquier, N. Polson, P. Rossi, Bayesian analysis of stochastic volatility models with fat-tails and correlated errors, J. Econometrics, (2004), forthcoming.

[9] A.C. Harvey, N. Shephard, The estimation of an asymmetric stochastic volatility model for asset returns, J. Business Econom. Statist. 14 (1996) 429-434.

[10] E. Jacquier, N. Polson, P. Rossi, Bayesian analysis of stochastic volatility models, J. Business Econom. Statist. 12 (1994) 371-389.

[11] S. Kim, N. Shephard, S. Chib, Stochastic volatility: likelihood inference and comparison with ARCH models, Rev. Econom. Studies 65 (1998) 361-393.

[12] N. Best, M. Cowles, K. Vines, Convergence diagnosis and output analysis software for gibbs sampling output version 0.30. MRC Biostatistics Unit, Institute of Public Health, 1999. 